\title{
Correlation between wetting properties and electrical performance of solution processed
}

\section{PEDOT:PSS/CNT nano-composite thin films}

\author{
Abdullah S Alshammari ${ }^{\text {a,b }}$, Maxim Shkunov and S Ravi P Silva ${ }^{\text {a* }}$ \\ ${ }^{a}$ Advanced Technology Institute, Faculty of Engineering and Physical Sciences, University of Surrey, \\ Guildford, UK, GU2 7XH \\ ${ }^{b}$ Department of Physics, College of Science, University of Hail, P.O.Box 2440, Hail, Saudi Arabia
}

\begin{abstract}
Nano-composite thin films of poly(3,4-ethylenedioxythiophene) poly(styrenesulfonate) (PEDOT:PSS) with different loading concentrations of Multi-walled carbon nanotubes (MWCNT) were deposited on glass substrates using inkjet printing and spin coating techniques. The surface energy of the substrate was modified using an oxygen plasma to achieve different degrees of wetting by the composite solution. We show that the electrical properties strongly depend on the wetting of the substrate and by controlling the wettability, the conductivity of the nano-composite samples can be improved. Based on polymer conductivity, the electrical conductivity of the composite film can be improved or degraded by orders of magnitude with the incorporation of the same concentration of MWCNT. Moreover, electrical measurements show strong correlation between the conductivity of the carbon nanotube network and the resulting nano-composite films. The dependence of electrical properties on the wettability and the conductivity of the composite components could explains the diversity in the electrical behaviour reported in the literature for PEDOT:PSS/MWCNT nano-composite thin films.
\end{abstract}

Keywords: Carbon nanotubes, PEDOT:PSS, Wettability, Electrical properties, Conductive nano-composite

\footnotetext{
"Corresponding author: s.silva@ surrey.ac.uk

Tel: +441483689825

Fax: +44 1483686081
} 


\section{Introduction:}

There is a considerable interest in using the conductive polymer PEDOT:PSS poly $(3,4-$ ethylenedioxythiophene) poly(styrene-sulfonate) in many electronic applications due to the properties this polymer offers such as high transparency in the visible range, high stability under ambient environments, mechanical flexibility and solution processability[1-3]. In addition, its high work function $(\sim 5.1 \mathrm{eV})$ makes it very effective hole transport layer in optoelectronic devices [4]. However, the moderate conductivity of PEDOT:PSS limits its use in a number of applications which require a metal-like conductivity. For this reason, intense efforts are on going to improve the conductivity of these polymers using different techniques. One of the most common routes used is adding conductive nano-materials such as carbon nanotubes to the polymer matrix. It has been shown that mixing carbon nanotubes with PEDOT:PSS improve the conductivity of the pristine polymer. The improvement in the electrical properties was attributed to the conductive nanotubes network which contributes to the conduction and also connects the conductive PEDOT islands in the composite film [5-8]. Contradictorily, some studies report a decrease in the conductivity of such composites after the addition of nanotubes and explain this in terms of the contact resistance between the nanotubes, the nanotubes and the polymer chains and the effect of the insulating functional groups used to disperse the nanotubes $[6,9,10]$. In addition, it can be noted from a number of studies on the electrical behaviour of PEDOT:PSS/CNT composite that the variation in the conductivity of the polymer after the addition of the nanotubes is within the intrinsic conductivity values of the polymer. The disparity in the reported results and the behaviour of these composites could be due to variations of the surface tension after adding nanotubes, conductivity of the pristine polymer and the conductivity of the percolated CNT network having not been fully considered. The effect of using additives, solvent and/or surfactant, such as glycerol, dimethyl sulfoxide (DMSO) and sorbitol on the surface tension of PEDOT:PSS solution and the electrical properties of the resulting films has been reported in many studies [11-14]. The change in the electrical properties is attributed to the morphological changes especially the roughness of the samples. These effects should also be involved in the case of PEDOT:PSS/CNT composites, since the addition of carbon nanotubes suspension to the conductive polymers is usually accompanied with a change in the surface tension of the composite solution which makes the comparison of the nano-composite and the pristine polymer 
conductivities invalid. Moreover, the types of CNT and polymer are very important and have significant effect on the percolation threshold and the conductivity of the resulting composite [15-19].

The purpose of this paper is to contribute to the research in the field of conducting nano-composite by further examination of the factors that affect the conductivity of the nano-composite samples. The paper shows the effect of wetting variations on the electrical properties of the printed polymer samples, and its impact to the overall electrical performance of the nano-composite thin films. Moreover, the paper presents the electrical performance of these composites when adding carbon nanotubes to different types of the conductive polymer PEDOT:PSS. We believe that these factors are of considerable significance and investigating their effects would allow for a better understanding of the performance of such composites.

\section{Experimental details}

In this study, the conductive polymer PEDOT:PSS (CLEVIOSTM P Jet HC and P Al 4083) was purchased from CLEVIOS. Multi-walled carbon nanotubes (MWCNT) (product no. 694185) were purchased from Sigma Aldrich. In order to mix CNT with the water based PEDOT:PSS ink, CNT were acid functionalized following the procedures reported by Hatton et al [20]. $250 \mathrm{mg}$ of MWCNTs were placed in a round bottom flask and mixed with 1:3 mixture of nitric acid and sulfuric acid using a sonication bath for 10 minutes to disperse the nanotubes with the acid mixture. Subsequently, the nanotube-acid mixture was refluxed at $130 \mathrm{oC}$ for 1 hour. Then, the mixture was diluted with deionized water and centrifuged for 10 minutes at $8500 \mathrm{rpm}$. The supernatant acid was removed and the remaining solid content washed with DIwater several times over a $0.2 \mu \mathrm{m}$ polycarbonate filter. In order to disperse CNTs in DI-water, the filter was immersed in DI-water and agitated for few minutes using a sonication bath. The dispersion was centrifuged three times at $8500 \mathrm{rpm}$ for 10,20 and 30 minutes respectively. Each time the supernatant was collected and centrifuged again until a black, clear and stable suspension was obtained. The MWCNT suspension was diluted with DI-water to obtain different concentration from 0.02 to $0.08 \mathrm{wt} \%$ and constant volume from each concentration was then added to the PEDOT:PSS solution in order to maintain the surface tension in all 
samples, including the pristine polymer. After adding nanotubes, each sample was sonicated for 30 minutes to obtain a homogeneous mixture. Samples were then deposited on glass substrates using two techniques: inkjet printing (Dimatix 2831 printer at driving voltage of $18 \mathrm{~V}$, drop spacing of $20 \mu \mathrm{m}$, jetting frequency of $5 \mathrm{KHz}$ and room temperature) and spin coating technique (at $2000 \mathrm{rpm}$ for $1 \mathrm{~min}$ ). To achieve different degrees of wetting of the substrate by the solution, substrates were cleaned by sonication for 5 minutes in acetone, isopropanol and methanol respectively. After each cleaning step the substrates were dried with nitrogen. Then the substrates were treated with oxygen plasma (RF power of 100 watts, $\mathrm{O}_{2}$ flow rate of $15 \mathrm{SCCM}$ and process pressure of $200 \mathrm{mT}$ Torr)for 1,5 and 10 minutes.

Scanning electron microscope (FEI Quanta 200) was used to image the samples with $5 \mathrm{kV}$ accelerating voltage and spot size of $3 \mathrm{~nm}$. A Veeco Dimension 3100 atomic force microscope was used in tapping mode to obtain the profile and thickness of the printed samples. Transmission line method (TLM) along with Keithley 4200 - SCS (semiconductor characterization system) were used to carry out electrical measurements. I-V curves were recorded over different length of the sample and the sheet resistance was obtained from the plot of resistance versus length. The contact angles were measured using Kruss-EasyDrop contact angle kit.

\section{Results and discussion}

Wetting properties can be evaluated by measuring the contact angle made by a liquid droplet on a solid surface. The contact angle is a measure of the spreading of a liquid on a solid surface and can be used to calculate the energetics of the solid-liquid, vapour-liquid and solid-vapour interfaces. The contact angle of a droplet at equilibrium is given by the following Young's relation [21]:

$$
\cos \theta_{Y}=\frac{\gamma_{s v-} \gamma_{s l}}{\gamma_{l v}}(1)
$$

Where $\theta_{Y}$ is Young contact angle, $\gamma_{v}$ is liquid-vapor surface tension, $\gamma_{s v}$ is solid-vapor surface tension and $\gamma_{s l}$ is solid-liquid surface tension. The images of PEDOT:PSS droplets on glass substrate are shown in figure 1. The contact angle of the droplet on solvent cleaned substrate (figure 1a) is about $13.3^{\circ}$ and decrease 
to about $6.1^{\circ}$ on 5 minutes - plasma treated substrate (figure 1c) which indicates the increase in the surface energy of the substrate and hence the improvement of wettability.

The impact of plasma treatment can also be observed from SEM images of the printed droplets where a significant change in the dried droplet shape occurred after the treatment. The figure also shows the effect of substrate temperature on the droplet shape as seen in (b) and (D). Furthermore, the figure illustrates the connection between the contact angle and the shape of the dried droplet where the less contact angle is the more spreading and the more contact area when the droplet dries.
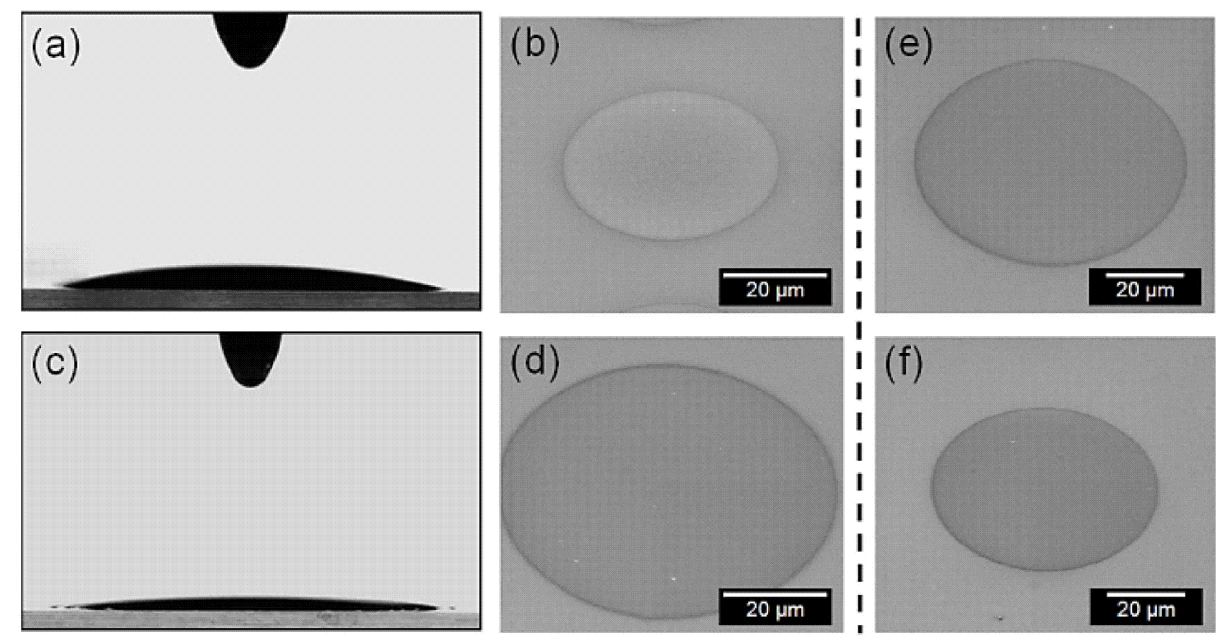

Fig. 1Effect of substrate treatment and temperature on droplet wetting: contact angle and SEM images of PEDOT:PSS droplet printed @ RT on (a,b) solvent cleaned and (c,d) plasma treated substrates. (e) and (f) are SEM images of printed PEDOT:PSS droplets on plasma treated substrate for 5 minutes and printed @ $40{ }^{\circ} \mathrm{C}$ and $60{ }^{\circ} \mathrm{C}$ respectively. 


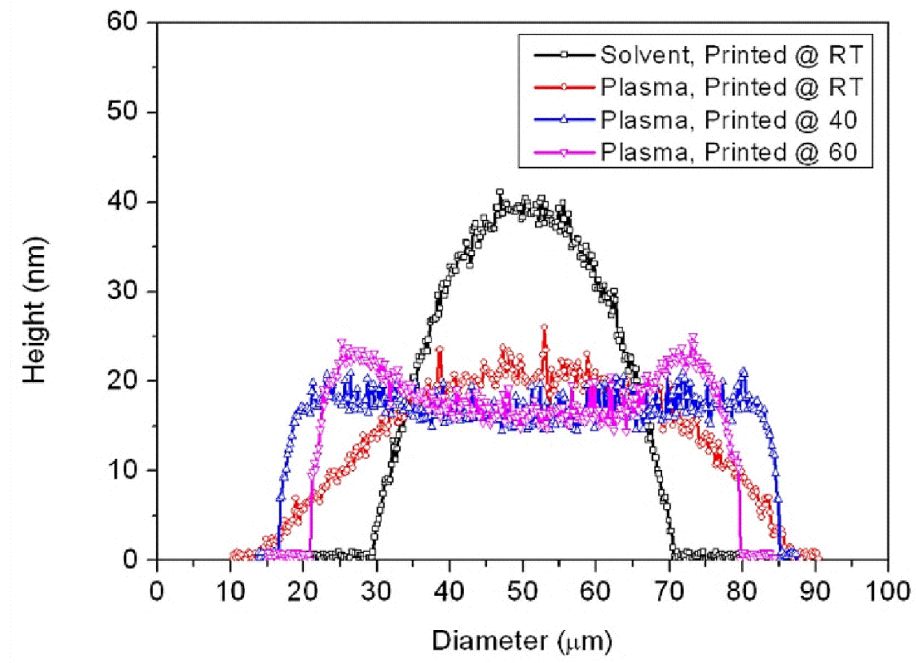

Fig. 2AFM-height profile of printed PEDOT:PSS droplets on glass substrates with different treatments and substrate temperatures.

The variation in the profile of the printed PEDOT:PSS droplets as a result of substrate treatment and temperature is illustrated in figure 2. It is observed in figure 2 that the printed droplet on solvent cleaned substrates is thicker with an average diameter of $41 \mu \mathrm{m}$. After plasma treatment, the wetting increases due to the increase in the surface energy of the substrate and a thinner droplet with large diameter $(\sim 74 \mu \mathrm{m})$ is produced. The figure also shows the effect of the substrate temperature on the printed droplets and it is noted that the increase in the temperature does not allow the droplets to spread on the surface due to the higher evaporation rate of the solvent, which causes the droplet to dry over a small area. Furthermore, it can be observed that increasing the substrate temperature activates the "coffee ring" effect and produces nonuniform droplets with spiky edges. To avoid this effect, all the samples were printed at room temperature. 

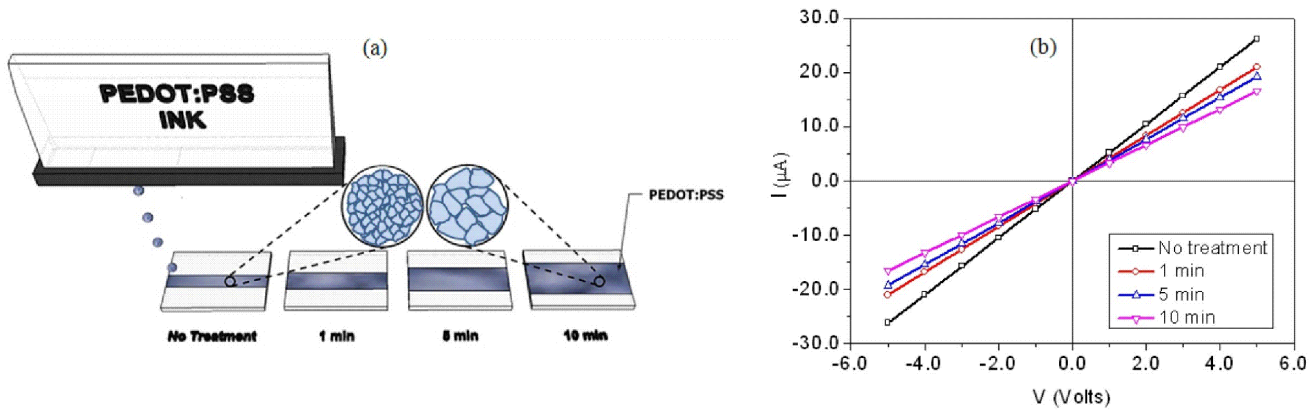

Fig. 3(a) Printing of PEDOT:PSS ink on four substrates with different surface energies at room temperature.

(b) I-V curves showing the effect of substrate treatment on the resistance of printed PEDOT:PSS samples.

To study the effect of ink wetting on the electrical properties, the surface tension of PEDOT:PSS inks needs to be modified by adding different solvents and/or different solvent quantities in order to achieve different degrees of wetting on the substrate. However, this approach may make the comparison more complicated as the effects of many other factors, beside the change in surface tension, needs to be considered. These include the ink viscosity, printing driving voltage, droplet volume and speed. Therefore, in order to make a suitable comparison and to limit the effects to the influence of wetting, PEDOT:PSS samples were printed on four substrates with different surface energies at the same time (same print run) with identical printing conditions. The substrates were solvent cleaned and plasma treated for 1,5 and 10 minutes and were maintained at room temperature during the printing process. A schematic of the printing process is shown in figure 3a. Figure 3 (b) shows the variation of electrical properties of the printed samples as a result of the change in the wetting properties. It can be seen from the figure that sample with no treatment (less wetting) shows the lowest resistance and the resistance increases as the treatment time increases (more wetting). The resistance values of the non-treated, 1, 5and 10 minutes plasma treated samples were found to be 191, 238, 260 and $302 \mathrm{k} \Omega$, respectively.It should be noted that although there are changes in the dimensions (mainly thickness and width) of the printed samples as a result of the variation in the wetting, the length and the crosssectional area remain the same. The cross-sectional area of the droplets was calculated by integrating the area under the profile curve in figure 2 and the average value was found to be $1.11 \mu \mathrm{m}^{2}$ with slight variation of \pm 
$0.04 \mu \mathrm{m}^{2}$ for the various droplets. To understand how the geometries affect the electrical properties of the structure, we consider the definition of resistance which is given as follows [22]:

$$
R=\rho(V / A)(2)
$$

Where $R$ is the resistance $(\Omega), \rho$ is the resistivity $(\Omega / \mathrm{cm}), l$ is the length $(\mathrm{cm})$ and A is the cross-sectional area $\left(\mathrm{cm}^{2}\right)$

Now as the length and cross-sectional area are the same for all the samples, the term $(I / A)$ in the right hand side of the equation should be constant. This suggests that the changes in the I-V curve and resistance should be directly related to the variations in the intrinsic resistivity of the samples. The variation in resistivity may be explained in term of the microstructural changes in the printed samples due to the differences in the degree of wetting of the droplet on the different substrates as shown in fig. 3 (a).

Figure 4 shows the AFM images of topography of the printed samples with different treatment. In the non-treated sample (less wetting), very good packing density is observed with small-stacked polymer grains. This leads to better overlap of the conductive chains, which enhances the conductivity of these samples. As the wetting increases with increasing substrate treatment time, droplets spread more and dry over larger areas and as a result, larger and more separated polymer islands are formed. This increases the distance between the conductive PEDOT particles, reduces their overlap and the probability of carrier hopping from one chain to another, resulting in the degradation of electrical conductivity. Moreover, it increases the thickness of the insulating PSS shell at the grain boundaries and makes carrier transport between the conductive islands less likely [23]. In addition to the microstructural changes, the surface roughness of the printed samples is found to increase as the ink wettability increases. The roughness increases from 0.96 to about $1.27 \mathrm{~nm}$ for the solvent cleaned and 10-mins plasma treated samples respectively. 
(a)
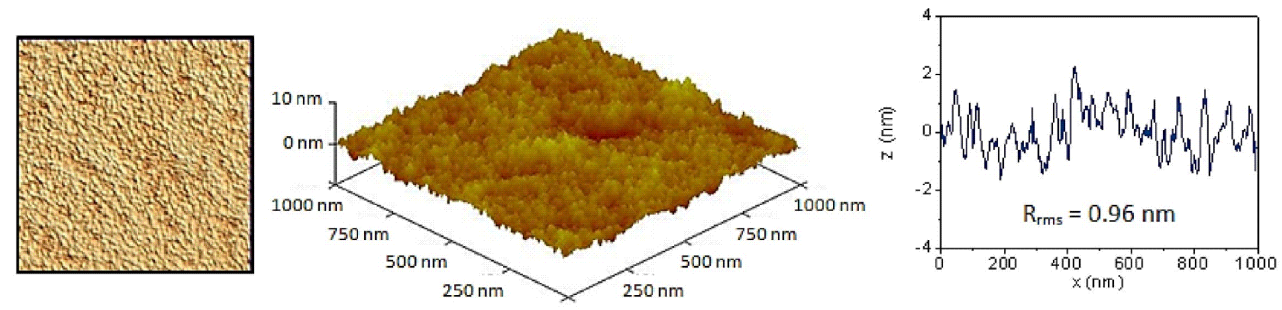

(b)
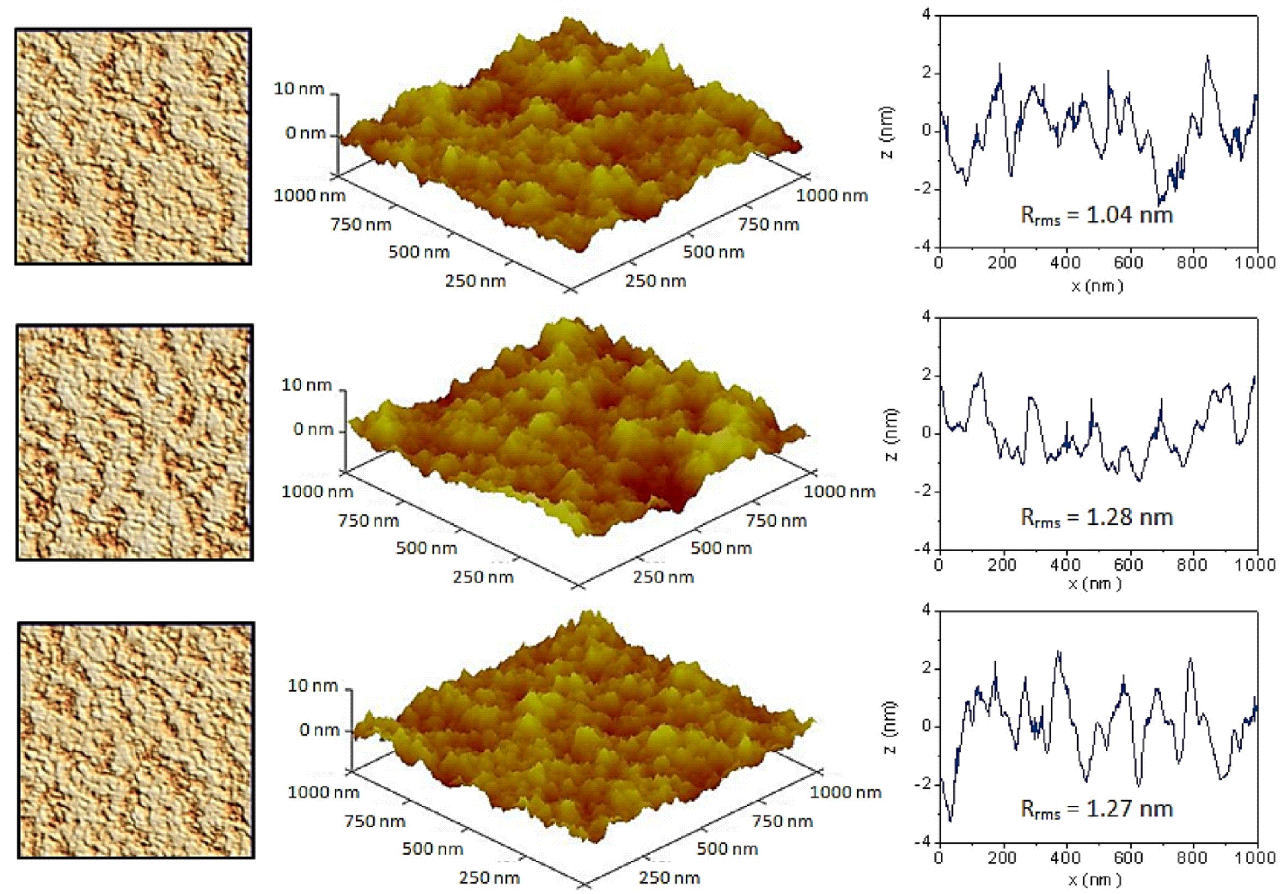

Fig. $41.0 \times 1.0 \mu \mathrm{m} 2 \mathrm{AFM}$ - phase images (Z-scale is $30^{\circ}$ ), 3D-height images and height profile with roughness values of printed samples on: (a) non- treated substrate and plasma treated substrates for (b) 1 , (c) 5 and (d) 10 minutes.

The change in the electrical conductivity of the polymer as a result of the variation in the wetting degree could help to understand the reasons behind the different observations on the electrical behaviour of PEDOT:PSS/CNT nano-composite reported in literature. The disparity in the results (regardless of an improvement or degradation in electrical performance) could be mainly due to the change in the wettability of 
the final composite solution, especially as the nanotubes are usually dispersed in a solution and then added to PEDOT:PSS. This has a significant impact on the surface tension and wettability of the composite solution and hence the microstructure and electrical properties. Moreover, the effect of the nanotubes on the morphology and structural disorders needs to be better understood. Table 1 shows some examples of sheet resistance values reported in the literature for PEDOT:PSS/CNT nano-composite.

Table 1. Conductivity of PEDOT:PSS/CNT composites reported in the literature.

\begin{tabular}{|c|c|c|c|c|c|}
\hline $\begin{array}{l}\text { PEDOT:PSS } \\
\text { Conductivity }\end{array}$ & $\begin{array}{c}\text { CNT } \\
\text { Concentration (wt. \%) }\end{array}$ & Technique & $\begin{array}{c}\text { Sheet Resistance } \\
\text { PEDOT:PSS }(\Omega / \square)\end{array}$ & $\begin{array}{c}\text { Sheet Resistance } \\
\text { PEDOT:PSS/CNT }(\Omega / \square)\end{array}$ & Ref. \\
\hline $\begin{array}{c}\text { CLEVIOS PH500 } \\
300 \mathrm{~S} / \mathrm{cm}\end{array}$ & $\begin{array}{c}\text { MWCNT } \\
1.47\end{array}$ & Spin coating & $\sim 980 \times 10^{3}$ & 734 & [5] \\
\hline $\begin{array}{c}\text { CLEVIOS PH500 } \\
300 \mathrm{~S} / \mathrm{cm}\end{array}$ & $\begin{array}{c}\text { SWCNT } \\
1\end{array}$ & Spin coating & 279 & 150 & [7] \\
\hline $\begin{array}{c}\text { CLEVIOS PH500 } \\
300 \mathrm{~S} / \mathrm{cm}\end{array}$ & $\begin{array}{c}\text { SWCNT } \\
0.01\end{array}$ & spray & 623 & 118 & [8] \\
\hline $\begin{array}{c}\text { Sigma-Aldrich } \\
1 \mathrm{~S} / \mathrm{cm}\end{array}$ & $\begin{array}{l}\text { SWCNT } \\
0.01\end{array}$ & Inkjet & $\sim 2000$ & 1000 & [6] \\
\hline $\begin{array}{l}\text { CLEVIOS P JET } \\
30-90 \mathrm{~S} / \mathrm{cm}\end{array}$ & $\begin{array}{l}\text { SWCNT } \\
0.5\end{array}$ & Inkjet & $\sim 1000$ & 225 & [10] \\
\hline $\begin{array}{l}\text { CLEVIOS P JET } \\
30-90 \mathrm{~S} / \mathrm{cm}\end{array}$ & $\begin{array}{c}\text { MWCNT } \\
0.1\end{array}$ & inkjet & $\sim 1000$ & 1904 & [10] \\
\hline
\end{tabular}

In fact, it can be noticed that most of the reported high conductivity values of PEDOT:PSS/CNT composites in literature are within the conductivity range of the pristine polymer. This suggests that, in many cases, the observed improvement in the electrical conduction of these composite may not be due to the incorporation of the nanotubes but merely the change in the surface tension as discussed previously and as a result the addition of the nanotubes is not justified. Furthermore, in many studies, the reported high conductivity results were achieved with highly conductive versions of the PEDOT:PSS and, therefore, it is important to consider the conductivity of the polymer and the conductivity of a distributed percolated network of the nanotubes when making such composites. The conductivity of a network of nanotubes is limited by the 
contact resistance at junctions between the nanotubes. The contact resistance between two nanotubes has been measured and found in the range of hundreds of $\mathrm{k} \Omega[24]$. This high contact resistance may prevent the nanotubes from contributing to the conduction process and also deteriorate the conductivity of the composite sample. However, the conductivity of CNT network can be significantly improved by increasing the length of the nanotubes, which assists to reduce the percolation threshold and reduce the number of nanotube junctions $[25,26]$.

To understand the effect of the polymer properties on the overall performance of the composite, we mixed carbon nanotubes with two different version of PEDOT:PSS, P Jet and P 4083. P Jet is a highly conductive polymer with conductivity values in the range of $30-90 \mathrm{~S} / \mathrm{cm}$, whereas $\mathrm{P} 4083$ conductivity varies from $2 \times 10^{-4}$ to $2 \times 10^{-3} \mathrm{~S} / \mathrm{cm}$. Figure 5 shows the morphology and electrical behaviour of these composites with different concentrations of the nanotubes. The SEM images in figure 5 show the samples before and after the incorporation of the nanotubes. In both cases, the samples show smooth featureless surface before adding the nanotubes. After the addition of the nanotubes, the images present good distribution of the nanotubes in both samples and confirm the formation of a percolated network of carbon nanotubes at 0.08 wt. \% concentration, which should have significant effect on the electrical properties of the polymer. It is observed from the electrical data in figure 5 that the influence of the nanotubes on the conductivity of the two composites is very different. Adding nanotubes to the highly conductive PEDOT:PSS version (P jet) affects the conductivity in an adverse manner although the good dispersion of the nanotubes in the sample. The sheet resistance values of the pristine PEDOT:PSS samples were found to be in the range of 300-500 $\Omega / \mathrm{sq}$ and increases for the composite films by about one order of magnitude with increasing CNT concentration from 0 to $0.08 \mathrm{wt} \%$. The degradation in the conductivity of the composite could be due to the increase in the structural disorder of the polymer chains and/or conducting grains after the incorporation of carbon nanotubes. The effect of disorder were studied and found to limits the conduction process in the conducting polymer PEDOT:PSS [27]. In the case of low conductivity PEDOT:PSS with non-treated substrate, the sheet resistance decreases from about $10^{8}$ to $10^{6} \Omega / \mathrm{sq}$ over the same concentration range of CNT. This corresponds to conductivity value of $0.17 \mathrm{~S} / \mathrm{cm}$ which is about two orders of magnitude higher than the intrinsic 
conductivity $\left(2 \times 10^{-3} \mathrm{~S} / \mathrm{cm}\right)$ of the pristine polymer and can be attributed to the presence of the nanotubes in the film. So, adding the nanotubes to this type of PEDOT:PSS enhances the conductivity of the resulting composite. It can be seen from the figure that at a concentration higher than $0.04 \%$ of CNT, the sheet resistance starts to decrease, which is indicative of the formation of a percolated conductive paths or networks within the film. This did not happen with the highly conductive PEDOT:PSS, although the same concentration of the nanotubes was used which support the important role of the polymer matrix. Another interesting observation from the figure is that the electrical percolation threshold in the composite with the lowly conductive PEDOT:PSS is shifted towards higher value of CNT concentration after plasma treatment. The sheet resistance starts to decrease for the samples with different plasma treatment times (more wetting) at a concentration of the nanotubes higher than $0.06 \mathrm{wt} . \%$. The shift of the percolation threshold can be attributed to the increase in the wettability which may disturb the nanotubes interconnection and as a result higher concentration of the nanotubes is required to form percolated conductive network. It is noteworthy that the sheet resistance of CNT film of similar thickness range without the polymer was also calculated and found to be $\sim 4 \times 10^{3} \Omega /$ sq. The variation in the sheet resistance values of the inkjet printed functionalized (oxidized) nanotubes as a function of the number of the printed layers/thickness was studied and is shown in figure 6 . The electrical measurements indicate that as the concentration of the nanotubes increase, the overall conductivity of the nano-composite samples appear to converge to a singular value for both types of PEDOT:PSS, with CNT. The convergent value could be that of the CNT network without any polymer matrix (i.e. $100 \mathrm{wt} \%$ concentration). 

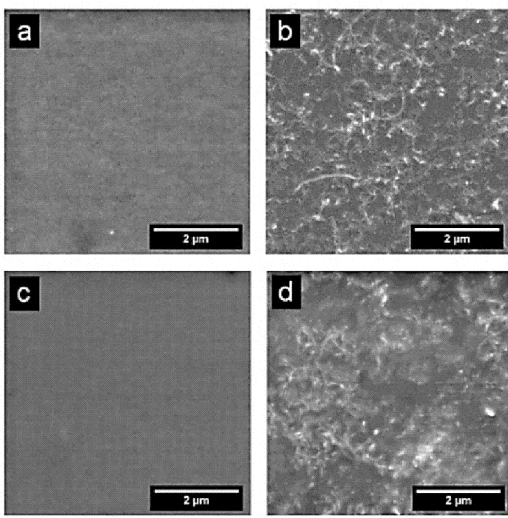

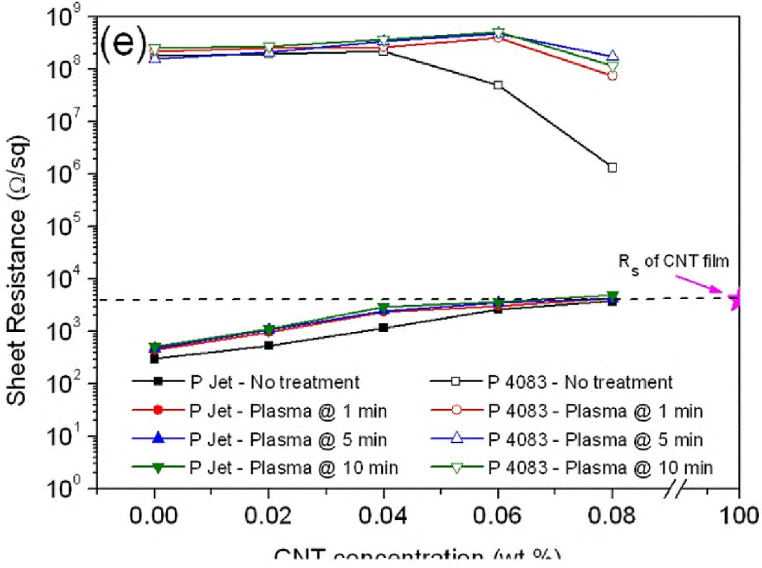

CNIT nnnennerntinn (mat \%

Fig. 5 SEM images of PEDOT:PSS/MWCNT samples: P Jet with (a) 0 wt.\% CNT, (b) 0.08 wt.\% CNT and P 4083 with (c) 0 wt.\% CNT, (d) 0.08 wt.\% CNT and (e) The effect of CNT concentration on the conductivity of two types of PEDOT:PSS with different treatment and the sheet resistance of CNT films (100 wt.\% concentration).

These results show that the conductivity of PEDOT:PSS/CNT nano-composite film depends on the conductivity of the pristine polymer on the one hand and on the conductivity of CNT percolated networks on the other. Furthermore, the best conductivity to be achieved for such a composite is limited by both the conductivity of the polymer and carbon nanotube network, whichever is higher. The reported improvements in the conductivity of highly conductive PEDOT:PSS versions after the addition of the nanotubes in some studies, therefore, could be attributed to the structural changes due to the change in the wetting properties and can be achieved by controlling the wettability of the ink even in the absence of the nanotubes, especially if the improvement is within the conductivity range of the pristine polymer. This highlights the importance of tuning the interaction between the ink and the substrate and how these factors could be utilized to produce samples with improved electrical conductivity. Moreover, the nanotube network needs to be optimised in terms of the nanotubes quality, their aspect ratio and dispersion, in order to make best use of their exceptional properties in improving the electrical performance of these conductive nano-composites. 


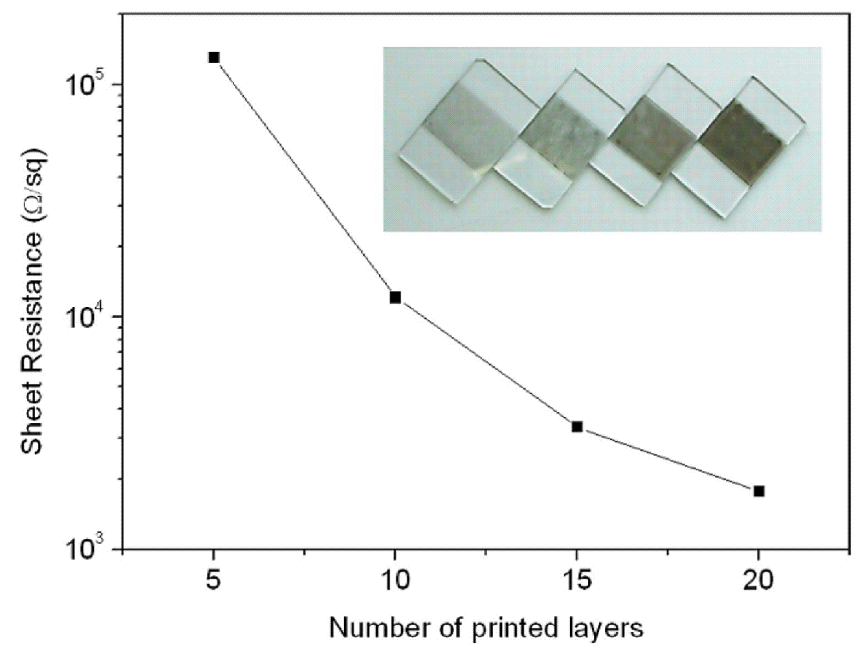

Fig. 6 Variation of the sheet resistance values of the printed carbon nanotubes with the number of the printed layers.

\section{Conclusions}

The effect of wetting properties on the electrical conductivity of PEDOT:PSS was studied and used to explain the observed variation in the performance of PEDOT:PSS/MWCNT nano-composite. The results show a significant effect of wetting on the conductivity of the films, which is mainly due to morphological changes induced by wettability variation. The changes in the surface tension of the nano-composite solution after the addition of the nanotubes could be the main factor behind the conductivity enhancement observed in many studies. In addition, the electrical performance of the composite was found to depend on the electrical properties of the conductive polymer and CNT network. Conductivity improvements by two orders of magnitude and degradation by one order were recorded after adding multi-walled carbon nanotubes to different types of PEDOT:PSS. Addition of carbon nanotubes to a conductive polymer matrix does not necessarily increase the conductivity of the resulting composite. The electrical properties of the initial materials used to make the conductive composites are of importance and dictate the electrical behaviour of such composites. The changes in the wetting properties and conductivity of the polymer and nanotube 
networks dictate the electrical performance of PEDOT:PSS/CNT, and should be considered when designing

and analysing the performance of nano-composite materials.

\section{Acknowledgment}

Mr Alshammari would like to acknowledge University of Hail and the Saudi Arabia cultural bureau for

financial support.

\section{References}

1. Agalya G, Lv C, Wang X, Koyama M, Kubo M, Miyamoto A (2005) Theoretical study on the electronic and molecular properties of ground and excited states of ethylenedioxythiophene and styrenesulphonic acid. Applied Surface Science 244 (1-4):195-198. doi:10.1016/j.apsusc.2004.09.139

2. Cruz-Cruz I, Reyes-Reyes M, Aguilar-Frutis MA, Rodriguez AG, López-Sandoval R (2010) Study of the effect of DMSO concentration on the thickness of the PSS insulating barrier in PEDOT:PSS thin films. Synthetic Met 160 (13-14):1501-1506. doi:10.1016/j.synthmet.2010.05.010

3. Kim WH, Kushto GP, Kim H, Kafafi ZH (2003) Effect of annealing on the electrical properties and morphology of a conducting polymer used as an anode in organic light-emitting devices. Journal of Polymer Science Part B-Polymer Physics 41 (21):2522-2528. doi:10.1002/polb.10646

4. Dimitriev OP, Grinko DA, Noskov YV, Ogurtsov NA, Pud AA (2009) PEDOT:PSS films-Effect of organic solvent additives and annealing on the film conductivity. Synthetic Met 159 (21-22):2237-2239. doi:10.1016/j.synthmet.2009.08.022

5. Yun DJ, Hong K, Kim S, Yun WM, Jang JY, Kwon WS, Park CE, Rhee SW (2011) Multiwall carbon nanotube and poly(3,4-ethylenedioxythiophene): polystyrene sulfonate (PEDOT:PSS) composite films for transistor and inverter devices. ACS applied materials \& interfaces 3 (1):43-49. doi:10.1021/am1008375

6. Mustonen T, Kordás K, Saukko S, Tóth G, Penttilä JS, Helistö P, Seppä H, Jantunen H (2007) Inkjet printing of transparent and conductive patterns of single-walled carbon nanotubes and PEDOT-PSS composites. physica status solidi (b) 244 (11):4336-4340. doi:10.1002/pssb.200776186

7. Park J, Lee A, Yim Y, Han E (2011) Electrical and thermal properties of PEDOT:PSS films doped with carbon nanotubes. Synthetic Met 161 (5-6):523-527. doi:10.1016/j.synthmet.2011.01.006

8. Zhang J, Gao L, Sun J, Liu Y, Wang Y, Wang J (2012) Incorporation of single-walled carbon nanotubes with PEDOT/PSS in DMSO for the production of transparent conducting films. Diamond and Related Materials 22:82-87. doi:10.1016/j.diamond.2011.12.008

9. Huh JW, Jeong JW, Lee JW, Shin S-I, Kwon J-H, Choi J, Yoon HG, Cho G-I, You I-K, Kang S-Y, Ju BK (2009) Carbon nanotube and conducting polymer dual-layered films fabricated by microcontact printing. Applied Physics Letters 94 (22):223311. doi:10.1063/1.3137185

10. Denneulin A, Bras J, Blayo A, Khelifi B, Roussel-Dherbey F, Neuman C (2009) The influence of carbon nanotubes in inkjet printing of conductive polymer suspensions. Nanotechnology 20 (38):385701. doi:10.1088/0957-4484/20/38/385701

11. Tekin E, Smith PJ, Schubert US (2008) Inkjet printing as a deposition and patterning tool for polymers and inorganic particles. Soft Matter 4 (4):703-713. doi:10.1039/b711984d

12. Wilson P, Lekakou C, Watts JF (2012) A comparative assessment of surface microstructure and electrical conductivity dependence on co-solvent addition in spin coated and inkjet printed poly $(3,4-$ ethylenedioxythiophene):polystyrene sulphonate (PEDOT:PSS). Org Electron 13 (3):409-418. doi:10.1016/j.orgel.2011.11.011 
13. Montibon E, Lestelius M, Jarnstrom L (2010) Electroconductive Paper Prepared by Coating with Blends of Poly(3,4-ethylenedioxythiophene)/Poly(4-styrenesulfonate) and Organic Solvents. Journal of Applied Polymer Science 117 (6):3524-3532. doi:10.1002/app.32250

14. Jönsson SKM, Birgerson J, Crispin X, Greczynski G, Osikowicz W, Denier van der Gon AW, Salaneck WR, Fahlman M (2003) The effects of solvents on the morphology and sheet resistance in poly $(3,4-$ ethylenedioxythiophene)-polystyrenesulfonic acid (PEDOT-PSS) films. Synthetic Met 139 (1):1-10. doi:10.1016/s0379-6779(02)01259-6

15. Bauhofer W, Kovacs JZ (2009) A review and analysis of electrical percolation in carbon nanotube polymer composites. Composites Science and Technology 69 (10):1486-1498. doi:10.1016/j.compscitech.2008.06.018

16. Sandler JKW, Kirk JE, Kinloch IA, Shaffer MSP, Windle AH (2003) Ultra-low electrical percolation threshold in carbon-nanotube-epoxy composites. Polymer 44 (19):5893-5899. doi:10.1016/s00323861(03)00539-1

17. Li J, Ma PC, Chow WS, To CK, Tang BZ, Kim J-K (2007) Correlations between percolation threshold, dispersion state, and aspect ratio of carbon nanotubes. Advanced Functional Materials 17 (16):3207-3215. doi:10.1002/adfm.200700065

18. Spitalsky Z, Tasis D, Papagelis K, Galiotis C (2010) Carbon nanotube-polymer composites: Chemistry, processing, mechanical and electrical properties. Progress in Polymer Science 35 (3):357-401. doi:10.1016/j.progpolymsci.2009.09.003

19. Moon JS, Park JH, Lee TY, Kim YW, Yoo JB, Park CY, Kim JM, Jin KW (2005) Transparent conductive film based on carbon nanotubes and PEDOT composites. Diamond and Related Materials 14 (11-12):18821887. doi:10.1016/j.diamond.2005.07.015

20. Hatton RA, Blanchard NP, Tan LW, Latini G, Cacialli F, Silva SRP (2009) Oxidised carbon nanotubes as solution processable, high work function hole-extraction layers for organic solar cells. Org Electron 10 (3):388-395. doi:10.1016/j.orgel.2008.12.013

21. Kwok DY, Neumann AW (1999) Contact angle measurement and contact angle interpretation. Advances in Colloid and Interface Science 81 (3):167-249. doi:10.1016/s0001-8686(98)00087-6

22. Sze SM (1985) Semiconductor Devices Physics and Technology. John willy \& sons, New York

23. Nardes AM, Kemerink M, Janssen RAJ, Bastiaansen JAM, Kiggen NMM, Langeveld BMW, van Breemen AJJM, de Kok MM (2007) Microscopic understanding of the anisotropic conductivity of PEDOT : PSS thin films. Advanced Materials 19 (9):1196. doi:10.1002/adma.200602575

24. Li C, Thostenson ET, Chou T-W (2008) Sensors and actuators based on carbon nanotubes and their composites: A review. Composites Science and Technology 68 (6):1227-1249. doi:10.1016/j.compscitech.2008.01.006

25. Hecht D, Hu L, Gruner G (2006) Conductivity scaling with bundle length and diameter in single walled carbon nanotube networks. Applied Physics Letters 89 (13). doi:10.1063/1.2356999

26. Simien D, Fagan JA, Luo W, Douglas JF, Migler K, Obrzut J (2008) Influence of nanotube length on the optical and conductivity properties of thin single-wall carbon nanotube networks. Acs Nano 2 (9):1879-1884. doi:10.1021/nn800376x

27. Sangeeth CSS, Jaiswal M, Menon R (2009) Correlation of morphology and charge transport in poly(3,4ethylenedioxythiophene)-polystyrenesulfonic acid (PEDOT-PSS) films. Journal of Physics-Condensed Matter 21 (7). doi:10.1088/0953-8984/21/7/072101 